\title{
The simulation of a two Dimensional Fokker Planck Equation for an Energetic Alpha Particle Beam in a Deuterium-Tritium Plasma using the Fokker Planck Package (FPPAC 81).
}

\author{
Nura, A.M. \\ Department of Physics, Bayero University, Kano.
}

\begin{abstract}
The two dimensional Fokker Planck equation is used to describe the time evolution of energetic beam of ions under the effect of dynamical friction and diffusion in velocity space, in a background plasma. The equation is simulated using the Fokker Planck package (FPPAC 81) to obtain the slowing down distribution function for energetic alpha particles which are produced continuously during deuterium-tritium fusion. The effect of varying the temperature for a given injection current which is taken to be a function of the reaction rate of the D-T fusion was studied. It was found that as the temperature increases from 10-20keV, the population of low energy particles decreases while that of high energy particles increases. The slowdown of alpha particles energy with time was faster as the temperature of the plasma increases. The energy increment of the D-T plasma and the electrons as the result of alpha particle heating was computed using the subroutine ENTRNS(k) in the FPPAC. The slowing down of the energetic alpha particle with time and the energy increment given to the plasma was found to be in good comparisons to that of a $180 \mathrm{keV}$ deuterium beam in a $5 \mathrm{keV}$ tritium plasma from literature. The energy increment for the plasma deuterium and tritium was found to decrease with temperature due to decrease in dynamical friction as a result of increase in the critical energy $W_{c}$.
\end{abstract}

Key words: Dynamical Friction, Diffusion, Distribution function, Critical Velocity, Injection Current, Reaction Rate.

\section{Introduction}

A situation that arises in many naturally occurring plasmas as well as fusion plasmas is that of a beam of fast ions moving through plasma. The beam ion energy is typically much larger than the temperature of the background plasma, i.e the velocity of the beam $V_{b}$ is greater than $V$ the velocity of the background ion velocity, but less than $V_{e}$ the background electron velocity. The beam ion may be the same type as the background ion or different. themselves.

In fusion research the plasma is self-heated by the energetic ions produced during the fusion reactions

$$
{ }_{1}^{2} \mathrm{D}+{ }_{1}^{3} \mathrm{~T} \rightarrow{ }_{2}^{4} \mathrm{He}(3.51 \mathrm{MeV})+{ }_{0}^{1} n(14.6 \mathrm{MeV})
$$

The alpha particles produced in the reaction above have energy 200 times that of the background plasma ion and are born with an isotropic distribution of velocities, and thermalizes with the background plasma particles as a result of multiple Coulomb collisions. In this work the thermalisation process is simulated by using the Fokker Planck Package (FPPAC81) to solve the Fokker Planck equation (FPE) for the beam ions, as it moves through the background plasma composed of Maxwellian ions and electrons with densities $n_{i}, n_{e}$, much greater than $n_{b}$, the beam ion density, in which Coulomb collisions of the beam ions with the background plasma results in the frictional drag on the background ions and electrons which cause the beam ions to slow down, and angular scattering on the background ions which cause the beam ions to be deflected from their original direction. The energy increment given to the plasma is also computed using the subroutine ENTRNS(k) from FPPAC. Section 2 comprises of the Fokker Planck Theory, section 3 describes the Methodology, section 4 consists of Results, and section 5 comprises of the Discussions and Conclusion.

\section{Theory}

Collisional interactions in a fully ionized plasma are predominantly due to the cumulative effects of many small angle deflections rather than due to a few large angle deflections. A formulation for describing the effects of multiple small angle Coulomb collisions on the distribution function $f(\vec{v})$ is the Boltzmann's kinetic equation with a collision term, known as Fokker Planck equation[1].

$$
\frac{\partial f_{a}}{\partial t}+\vec{v} \frac{\partial f_{a}}{\partial r}+\frac{F_{a}}{m_{a}} \frac{\partial f_{a}}{\partial v}=\left(\frac{\partial f_{a}}{\partial t}\right)_{\text {coll }}+S_{a}+L_{a}
$$

Where $f_{a}$, is the distribution function of the beam ion, $F_{a}$ is the force, $\left(\frac{\partial f_{a}}{\partial t}\right)_{\text {coll }}$ is the collision term, $S_{a}$ is the source term, and $L_{a}$ the loss term.

The collision term is given by[2,3] 


$$
\left(\frac{\partial f_{a}}{\partial t}\right)_{\text {coll }}=-\frac{\partial}{\partial v}[\langle\Delta v\rangle f(v, t)]+\frac{1}{2} \frac{\partial^{2}}{\partial v_{i} \partial v_{k}}\left[\left\langle\Delta v_{i} \Delta v_{k}\right\rangle f(v, t)\right]
$$

Equation (3) was formulated by A.D. Fokker and M. Planck to treat Brownian motion [2,3]. It describes the evolution in time of the distribution function due Coulomb collisions. Where the magnitude of $\langle\Delta v\rangle$ is the dynamical friction giving rise to a slowing down of the directed motion of the particles, and $\left\langle\Delta v_{i} \Delta v_{k}\right\rangle$ are velocity diffusion coefficient's and their effect is to bring about spreading of the particles velocity over a wider region of velocity space. The competition between the dynamical friction and velocity diffusion gives rise to the Maxwellian distribution in steady state.

A modification of the formulation of the Fokker-Planck equation derived by Rosenbluth et al [3] is

$$
\begin{aligned}
& \langle\Delta v\rangle_{a}=\Gamma_{a} \frac{\partial h_{a}}{\partial v}, \\
& \left\langle\Delta v_{\mathrm{i}} \Delta v_{\mathrm{k}}\right\rangle_{a}=\Gamma_{\mathrm{a}} \frac{\partial^{2} \mathrm{~g}_{\mathrm{a}}}{\partial v_{\mathrm{i}} \partial v_{\mathrm{k}}}
\end{aligned}
$$

So that the Fokker Planck operator becomes

$$
\left.\frac{\partial f}{\partial t}\right|_{\text {Coll }}=\Gamma_{a}\left\{\partial / \partial v_{i}\left(f_{a} \frac{\partial h_{a}}{\partial v_{i}}\right)+\frac{1}{2} \frac{\partial^{2}}{\partial v_{\mathrm{i}} \partial v_{\mathrm{k}}}\left(\mathrm{f}_{\mathrm{a}} \frac{\partial^{2} \mathrm{~g}_{\mathrm{a}}}{\partial v_{\mathrm{i}} \partial \mathrm{v}_{\mathrm{k}}}\right)\right\}
$$

Where the usual summation convention over repeated index $i$ and $j$ are used and where

$$
\Gamma_{\mathrm{a}}=\frac{4 \pi \mathrm{Z}_{\mathrm{a}}^{4} \mathrm{e}^{4}}{\mathrm{~m}_{\mathrm{a}}^{2}} .
$$

The Rosenbluth potentials are

$$
\begin{aligned}
& g_{a}=\sum_{b}\left(\frac{z_{b}}{z_{a}}\right)^{2} \ln \Lambda_{\mathrm{ab}} \int \mathrm{f}_{\mathrm{b}}\left(\mathrm{v}^{\prime}\right)\left|v-v^{\prime}\right| d v^{\prime} \\
& h_{a}=\sum_{b} \frac{m_{a}+m_{b}}{m_{b}}\left(\frac{z_{b}}{z_{a}}\right)^{2} \ln \Lambda_{\mathrm{ab}} \int \mathrm{f}_{\mathrm{b}}\left(\mathrm{v}^{\prime}\right)\left|v-v^{\prime}\right|^{-1} d v^{\prime}
\end{aligned}
$$

Here, the Coulomb logarithm, depends on both the interacting species, and is

$$
\ln \Lambda_{\mathrm{ab}}=\ln \left\{\left(\frac{\mathrm{m}_{\mathrm{a}} \mathrm{m}_{\mathrm{b}}}{\mathrm{m}_{\mathrm{a}}+\mathrm{m}_{\mathrm{b}}}\right)\left(\frac{2 a c \lambda_{\mathrm{D}}}{\mathrm{e}^{2}}\right) \max \left(\frac{2 \mathrm{E}_{\mathrm{k}}}{\mathrm{m}_{\mathrm{k}}}\right)_{\mathrm{a}, \mathrm{b}}^{1 / 2}\right\}-1 / 2
$$

where $\alpha=0.0073$ is the fine structure constant, $\quad \lambda_{D}=\sqrt{\frac{E_{e}}{6 \pi n_{e}^{2}}}$ is the Debye length

$n_{e}$ is the electron density and $E_{e}$ is the electron mean energy.

Equation (6) then becomes (in conservative form)

$$
\frac{1}{\Gamma_{a}}\left(\frac{\partial f_{a}}{\partial t}\right)=\frac{1}{v^{2}} \frac{\partial G_{a}}{\partial v}+\frac{1}{v^{2} \sin \theta} \frac{\partial H_{a}}{\partial \theta},
$$

Where

$$
\begin{aligned}
& G_{a}=A_{a} f_{a}+B_{a} \frac{\partial f_{a}}{\partial v}+C_{a} \frac{\partial f_{a}}{\partial \theta}, \\
& H_{a}=D_{a} f_{a}+E_{a} \frac{\partial f_{a}}{\partial v}+F_{a} \frac{\partial f_{a}}{\partial \theta} .
\end{aligned}
$$

The coefficients $A_{a}, B_{a}, C_{a}, D_{a}, E_{a}, F_{a}$ are given in [1,3]

As suggested by Rosenbluth et al [3], the "Rosenbluth potentials" and the distribution functions themselves may be represented by expansions in the Legendre polynomials.

$$
f_{a}(v, \theta, t)=\sum_{j=0}^{\infty} V_{j}^{b}(v, t) P_{j}(\cos \theta),
$$

Where, $V_{j}^{b}(v, t)=\frac{2 j+1}{2} \int_{-1}^{+1} f_{b}(v, \theta, t) P_{j}(\cos \theta) d(\cos \theta)$,

The expansions for the potentials are

$$
g_{a}(v, \theta, t)=\sum_{j=0}^{\infty} \sum_{b}\left(\frac{z_{b}}{z_{a}}\right)^{2} \ln \Lambda_{\mathrm{ab}} B_{j}^{b}(v, t) P_{j}(\cos \theta),
$$

and

$$
h_{a}(v, \theta, t)=\sum_{j=0}^{\infty} \sum_{b}\left(\frac{z_{b}}{z_{a}}\right)^{2}\left(\frac{m_{a}+m_{b}}{m_{b}}\right) \ln \Lambda_{\mathrm{ab}} A_{j}^{b}(v, t) P_{j}(\cos \theta) .
$$

With coefficients given by,

$$
\begin{gathered}
A_{j}^{b}(v, t)=\frac{4 \pi}{2 j+1}\left[\int_{0}^{v} \frac{\left(v^{\prime}\right)^{j+2}}{v^{j-1}} V_{j}^{b}(v, t) d v^{\prime}+\int_{v}^{\infty} \frac{v^{j}}{v^{j-1}} V_{j}^{b}(v, t) d v^{\prime}\right] \\
B_{j}^{b}(v, t)=-\frac{4 \pi}{4 j^{2}-1}\left[\int_{0}^{v} \frac{\left(v^{\prime}\right)^{j+2}}{v^{j-1}}\left(1-\frac{j-\frac{1}{2}\left(v^{\prime}\right)^{2}}{j+\frac{3}{2}(v)^{2}}\right) V_{j}^{b}(v, t) d v^{\prime}+\int_{v}^{\infty} \frac{v^{j}}{v^{j-3}}\left(1-\frac{j-\frac{1}{2}(v)^{2}}{j+\frac{3}{2}(v)^{2}}\right) V_{j}^{b}\left(v^{\prime}, t\right) d v^{\prime}\right] .
\end{gathered}
$$

2.1 Integration

Trapezoidal integration is used, such that the density of species a is given by

$$
n_{a}=2 \pi \iint f(v, \theta) v^{2} \sin \theta d \theta d v=2 \pi \sum_{i, j} a_{j} b_{i} f_{a}\left(v_{j}, \theta_{i}\right),
$$


and the energy density of species is

$$
n_{a} E_{a}=\pi m_{a} \sum_{i, j} a_{j} b_{i} v_{j}^{2} f_{a}\left(v_{j}, \theta_{i}\right)
$$

\subsection{Spatial Differencing}

The spatial derivatives are discretised using central finite difference as follows

$$
\begin{aligned}
& \frac{\partial}{\partial v}(A f)_{i, j} \approx\left(A_{i, j+1} f_{i, j+1}-A_{i, j-1} f_{i, j-1}\right) / 2 \Delta v_{j}, \\
& \frac{\partial}{\partial v}\left(B \frac{\partial}{\partial v} f\right)_{i, j} \approx\left\{\frac{B_{i, j+1 / 2}\left(f_{i, j+1}-f_{i, j}\right)}{\Delta v_{j+1 / 2}}-\frac{B_{i, j-1 / 2}\left(f_{i, j}-f_{i, j-1 / 2}\right)}{\Delta v_{j-1 / 2}}\right\} / \Delta v_{j}, \\
& \frac{\partial}{\partial v}\left(C \frac{\partial}{\partial \theta} f\right)_{i, j} \approx\left\{\frac{c_{i, j+1}\left(f_{i+1, j+1}-f_{i-1, j}+1\right)}{2 \Delta \theta_{i}}-\frac{B_{i, j-1}\left(f_{i+1, j-1}-f_{i-1, j-1}\right)}{2 \Delta \theta_{i}}\right\} / 2 \Delta v_{j},
\end{aligned}
$$

Where

$\Delta v_{j \pm 1 / 2}= \pm\left(v_{j+1} \pm v_{j}\right), \Delta v_{j}=\left(v_{j+1}-v_{j-1}\right) / 2, \Delta \theta_{i}=\left(\theta_{i+1}-\theta_{i-1}\right) / 2 \quad$,

$\Delta \theta_{i \pm 1 / 2}= \pm\left(\theta_{i \pm 1}-\mp \theta_{i}\right), B_{i, j \pm 1 / 2}=\left(B_{i, j \pm 1}+B_{j}\right) / 2$

Similarly for $\theta$ direction.

\subsection{Time Discretisation}

The collision operator is time integrated using alternating direction implicit (ADI), or fully. The procedure for the (ADI) $[4,5]$ is given as:

$$
\begin{aligned}
& Q^{n}\left(\frac{f_{i, j}^{n+1 / 2}-f_{i, j}^{n}}{\Delta t / 2}\right)=\frac{1}{v^{2}} \frac{\delta}{\delta v}\left(A^{n} f_{i, j}^{n+1 / 2}+B^{n} \frac{\delta f_{i, j}^{n+1 / 2}}{\delta v}+C^{n} \frac{\delta f_{i, j}^{n+1 / 2}}{\delta \theta}\right) \\
& \quad+\frac{1}{v^{2} \sin \theta} \frac{\delta}{\delta \theta}\left(D^{n} f_{i, j}^{n}+E^{n} \frac{\delta f_{i, j}^{n}}{\delta v}+F^{n} \frac{\delta f_{i, j}^{n}}{\delta \theta}\right)+K^{n} f_{i, j}^{n+1 / 2}+J^{n} \\
& Q^{n}\left(\frac{f_{i, j}^{n+1}-f_{i, j}^{n+1 / 2}}{\Delta t / 2}\right)= \frac{1}{v^{2}} \frac{\delta}{\delta v}\left(A^{n} f_{i, j}^{n+1 / 2}+B^{n} \frac{\delta f_{i, j}^{n+1 / 2}}{\delta v}+C^{n} \frac{\delta f_{i, j}^{n+1 / 2}}{\delta \theta}\right) \\
&+\frac{1}{v^{2} \sin \theta} \frac{\delta}{\delta \theta}\left(D^{n} f_{i, j}^{n+1}+E^{n} \frac{\delta f_{i, j}^{n+1}}{\delta v}+F^{n} \frac{\delta f_{i, j}^{n+1}}{\delta \theta}\right)+K^{n} f_{i, j}^{n+1}+J^{n}
\end{aligned}
$$

The difference equations (24), and (25) and the boundary conditions written in the tridiagonal form are

$$
\begin{aligned}
& -\alpha_{i, j}^{n} f_{i, j+1}^{n+1 / 2}+\beta_{i, j}^{n} f_{i j}^{n+1 / 2}-\gamma_{i, j}^{n} f_{i, j-1}^{n+1 / 2}=\delta_{i, j}^{n} \\
& -\varepsilon_{i, j}^{n} f_{i, j+1}^{n+1}+\mu_{i, j}^{n} f_{i j}^{n+1}-v_{i, j}^{n} f_{i, j-1}^{n+1}=\delta_{i, j}^{n}
\end{aligned}
$$

Where $\alpha, \beta, \gamma, \varepsilon, \mu, \nu$ are known quantities. The procedure for solving eqs. (26) and (27) is the standard technique for solving tridiagonalsystems[5].

\subsection{Analytical Beam Distribution Function}

For an energetic beam of particles that are generated during fusion such as the alpha particles, the distribution function is given by $[6,7]$

$$
f(V)=\frac{S \epsilon_{0}^{2} M M_{b}}{n_{e} Z Z_{b}^{2} e^{4} \ln \Lambda}\left(\frac{1}{1+\frac{V^{3}}{V_{c}^{3}}}\right)
$$

Where $S=n_{D} n_{T}\langle\sigma V\rangle_{D T}$ is the alpha particle injection current, and

where,

$V_{c}=\left[3^{\frac{1}{3}} Z^{\frac{1}{3}}\left(\frac{\pi}{2}\right)^{\frac{1}{6}}\right]\left[\frac{T_{e}}{\left(m^{\frac{1}{3}} M^{\frac{2}{3}}\right)}\right]^{\frac{1}{2}}$ is the critical velocity of the beam particle( the velocity at which the rate of energy lost by the beam to the plasma ions and electrons is equal). $n_{D}, n_{T}$, are the deuterium and tritium ion densities, $\langle\sigma V\rangle_{D T}$ is the reaction rate parameter, $n_{e}$ is the electron density.Zand $Z_{b}$ are the charge numbers for the plasma and beam ions, $M a n d M_{b}$ are the mass numbers for the plasma and beam ions, $m$ and $T_{e}$ are the mass and temperature of the electron respectively.

\subsection{Slowing Down Time}

The slowing down time for an ion in the plasma at temperature $\mathrm{T}_{\mathrm{e}}$ is given by [7]

$$
\tau_{s}=0.012 \frac{\left(T_{e}[\mathrm{keV}]\right)^{3 / 2} \mathrm{M}_{b}}{n_{e}\left[10^{20} \mathrm{~m}^{-3}\right] Z_{b}^{2}}
$$

and the critical energy $W_{c}=1 / 2 m_{b} v_{c}^{2}=14.8 T_{e} M_{b}\left(\frac{1}{n_{e}} \frac{\sum_{i} n_{i} Z_{i}^{2}}{M_{i}}\right)^{2 / 3}$ 


\subsection{The Rate Of Energy Transfer And Energy Increment}

The rate of energy transfer to the plasma ions and electrons is obtained from the package as "ENTRA" , in $\mathrm{keV} \mathrm{cm}^{-3} \mathrm{~s}^{-1}$ and the energy increment $\Delta \mathrm{W}$ is given as [2],

$$
\Delta W=\frac{E N T R A}{J_{\alpha}},
$$

where $J_{\alpha}$ is the alpha particle injection current density.

\subsection{Fokker Planck Package}

\section{Methodology}

The package was written by McCoy, Mirin, and Killeen [1] and solves the Fokker-Planck equations for an arbitrary number of charged species, described by distribution functions of speed $v$ and pitch angle $\theta$ in the presence of arbitrary number of fixed Maxwellian species. It comprises of two main parts. The subroutine COEF which is used to compute the Fokker Planck coefficientsand subroutine XSWEEP which is used to time integrate the distribution functions using either implicit operator splitting or an alternating direction implicit (ADI) method. The driver calls the subroutine INITIAL which reads the input data, initialize certain package and driver variables and call several subroutines, such as SETPARS which copies values in parameter statement into common storage, XINIT for setting up the spatial mesh, XINITL for computing constants and constant arrays, FINIT which initializes the distribution functions, SOURCE for calculating the sources in velocity space, GNANDE for computing the densities, and energies. The initialization ends with a call to EEPRINT which print the distribution function and other information. The driver then solves the Fokker-Planck operator for NSTOP time steps, by calls to three package routines, SETITUP, COEF, and XSWEEP. Where, subroutine SETITUP in turn calls PREPKG1 for setting the boundary conditions, GNANDS for copying densities and energies into package arrays, while GAMMAI computes the Coulomb logarithm. After the new distribution are calculated, the new densities and energies are computed for all species in GNANDE. This is repeated for NSTOP time steps, and output is given for NPRINT times.

\subsection{Procedure}

An input file named alpha2beam.dat was created, where an alpha particle of mass number $M_{b}=4$, charge number $Z_{b}=2$, injection energy $E_{b}=3.50 \mathrm{MeV}$, injection current $S=n_{D} n_{T}\langle\sigma V\rangle_{D T}$, where $\langle\sigma V\rangle_{D T}=$ $4.2 \times 10^{-22} \mathrm{~m}^{3} \mathrm{~s}^{-1}$, was injected into a D-T plasma with $n_{T}=n_{D}=5 \times 10^{19} \mathrm{~m}^{-3}, n_{e}=10^{20} \mathrm{~m}^{-3}, T_{e}=T_{i}=$ 10,15 , and $20 \mathrm{keV}$. The ion species in the plasma was considered to be the average of $M_{D}$ and $M_{T}$ i.e $M_{D T}=$ 2.5, $Z_{D T}=1.0, n_{D T}=10^{20} \mathrm{~m}^{-3}$. The slowing down distribution for the energetic alpha particle beam was obtained from the FPPAC. The distribution function for the alpha particle was also obtained from the distribution given in Equation (28). A comparison was made between the two distributions at different values of $T_{e}$. The Slowing down of energy of the alpha particle beam in the plasma at $T_{e}=T_{i}=10,15$, and $20 \mathrm{keV}$ was also simulated. The energy increment $\Delta W_{D T}$ and $\Delta W_{e}$ given to the plasma deuterium-tritium ions and the electrons was computed from the subroutine ENTRNS $(\mathrm{k})$ using the injection current $J_{\alpha}=S$. The procedure was repeated for different values of plasma temperature. A comparison was made to the slowing down of a $180 \mathrm{keV}$ deuterium beam in a $5 \mathrm{keV}$ plasma obtained from the literature [6]

\subsection{Slowing down distribution function}

\section{Results}

The graph of the slowing down distribution of energetic alpha particle beam as a function of normalized velocity is shown in figures $1-3$ for varying plasma temperatures $T=10-20 \mathrm{keV}$. The population of low energy ions in the alpha particle beam is seen to decrease with temperature. The computed distribution function which did not neglect angular dependence compares fairly well with the calculated slowing down distribution which neglects the pitch angle dependence[6,7] 


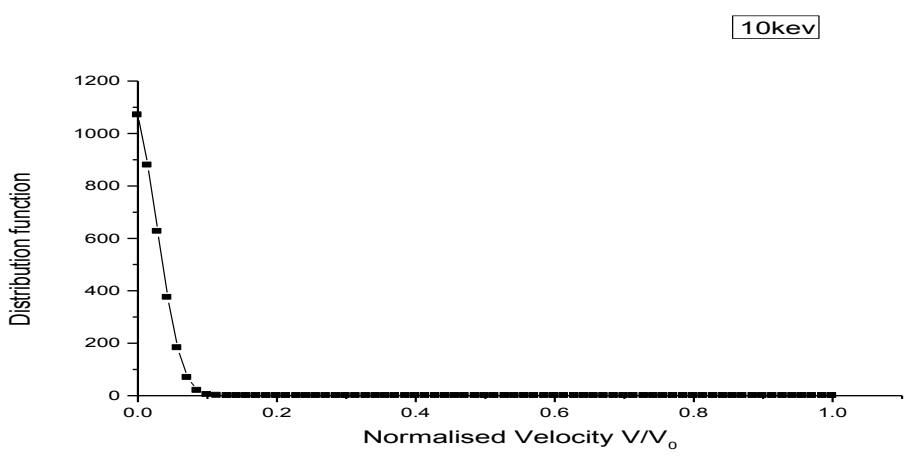

Figure 1 The distribution function for $3.5 \mathrm{MeV}$ alpha particle beam in a $10 \mathrm{keV}$ deuterium-tritium plasma against the normalised velocity. The population of low energy particles is very high while that of high energy particles is low. Most of the beam energy is transferred to the plasma when $\mathrm{V}$ is less than $0.3 \mathrm{~V}_{0}=\mathrm{V}_{\mathrm{c}}$

$15 \mathrm{kev}$

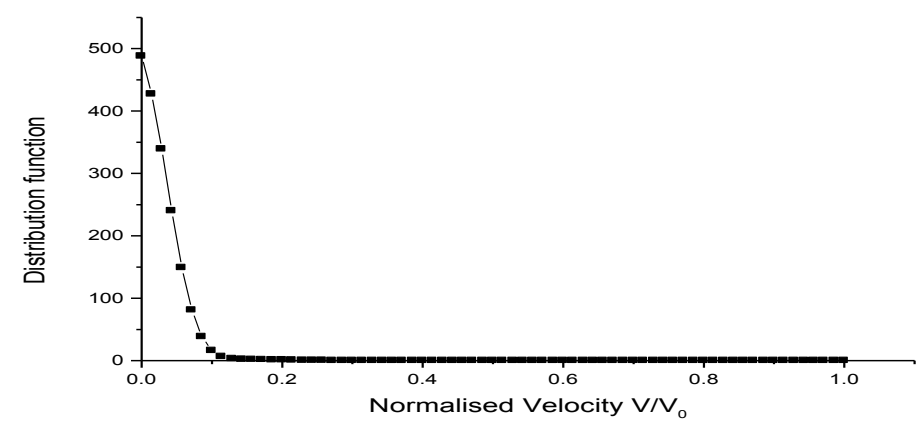

Figure 2 The distribution function for $3.5 \mathrm{MeV}$ alpha particle beam in a $15 \mathrm{keV}$ deuterium-tritium plasma against the normalised velocity. The population of low energy particles is lower than that in a $10 \mathrm{keV}$ plasma very high while that of high energy particles has increased. Most of the beam energy is transferred to the plasma when $\mathrm{V}$ is less than $0.37 \mathrm{~V}_{0}=\mathrm{V}_{\mathrm{c}}$.

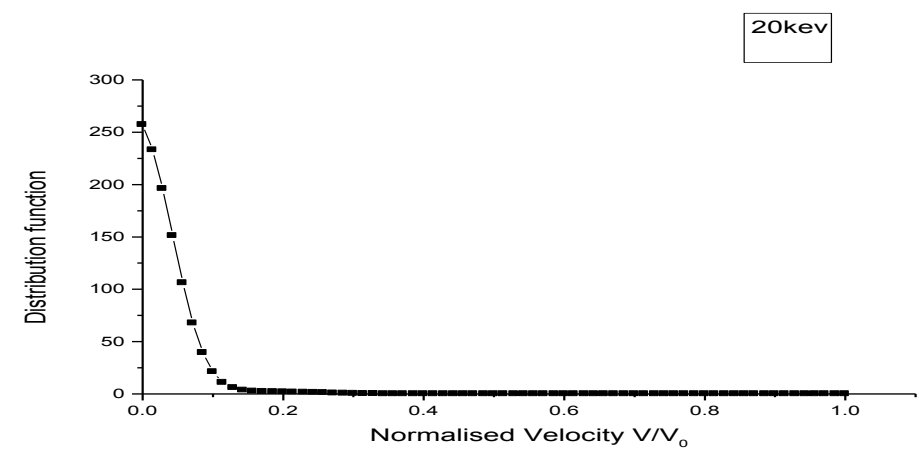

Figure3: The distribution function for $3.5 \mathrm{MeV}$ alpha particle beam in a $20 \mathrm{keV}$ deuterium-tritium plasma against the normalised velocity. The population of low energy particles is further reduced while that of high energy particles is increased further. Most of the beam energy is transferred to the plasma when $\mathrm{v}$ is less than $0.43 \mathrm{~V}_{0}=\mathrm{V}_{\mathrm{C}}$ 


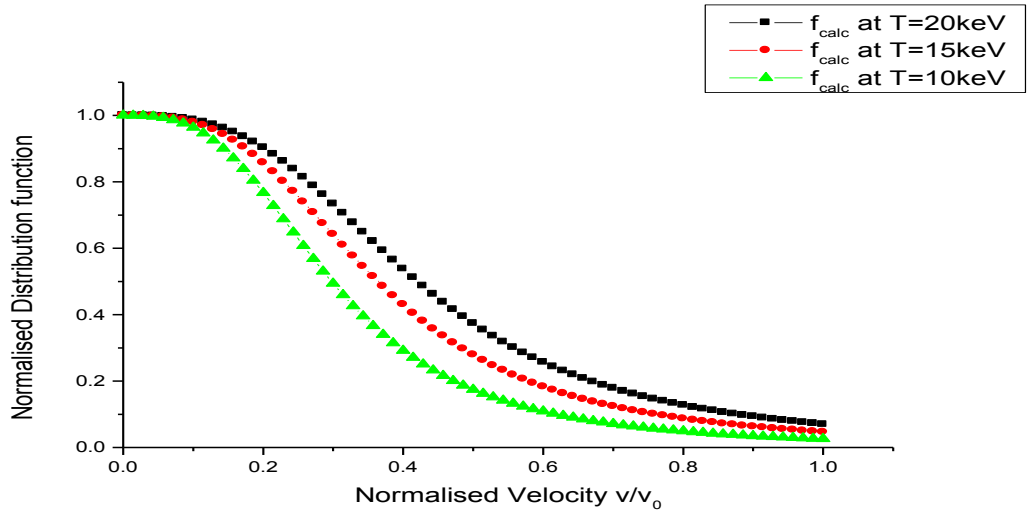

Fig 4The calculated normalised alpha particle beam Slowing down distribution functions, for Plasma at $\mathrm{T}=10$, 15 , and $20 \mathrm{keV}$. Using Equation (28)

\subsection{Slowing down of Energy}

Using FPPAC the slowdown of $3.5 \mathrm{MeV}$ alpha particle energy with time (normalised) to density was computed for plasma temperatures $\mathrm{T}_{\mathrm{e}}=10-20 \mathrm{keV}$

$\mathrm{T}_{\mathrm{e}}=10 \mathrm{keV}$

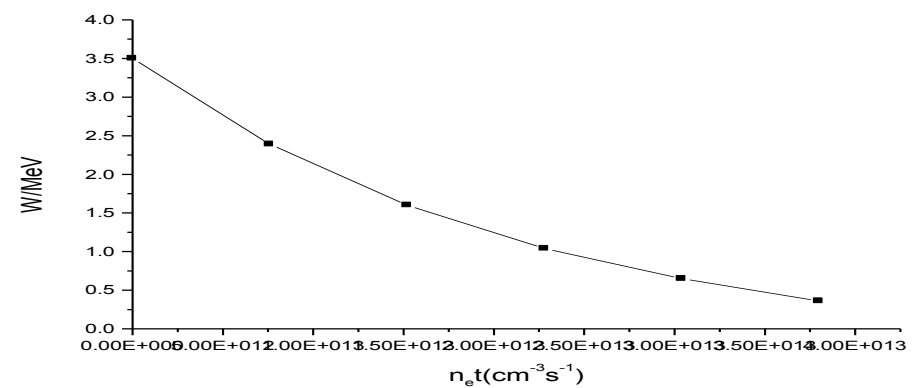

Figure5: The Slowing down of a $3.5 \mathrm{MeV}$ energetic alpha particle beam in a D-T plasma at $\mathrm{T}=10 \mathrm{keV}$ against normalised time, using FPPAC

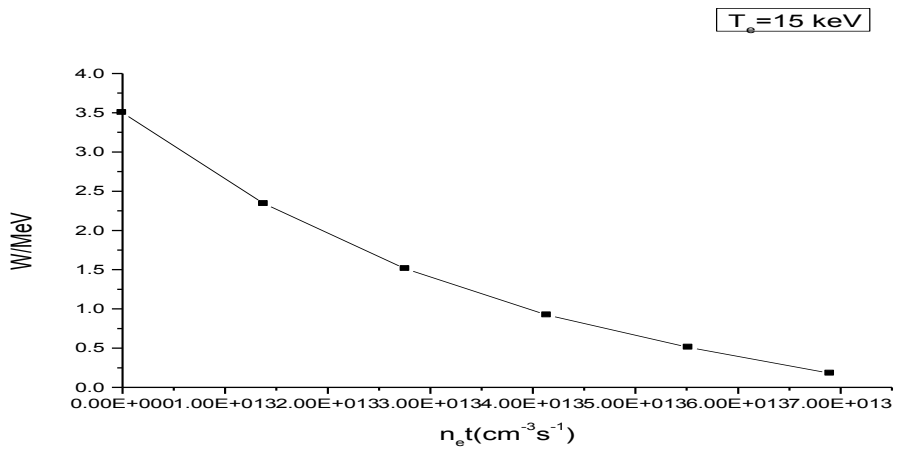

Figure6: The Slowing down of a $3.5 \mathrm{MeV}$ energetic alpha particle beam in a D-T plasma at T=15 keV against normalised time. , using FPPAC

$T_{e}=20 \mathrm{keV}$

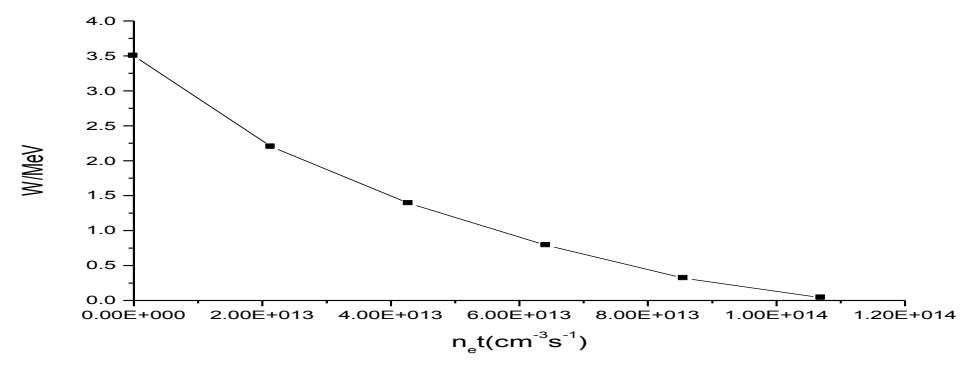


Figure7: The Slowing down of a $3.5 \mathrm{MeV}$ energetic alpha particle beam in a D-T plasma at $\mathrm{T}=20 \mathrm{keV}$.

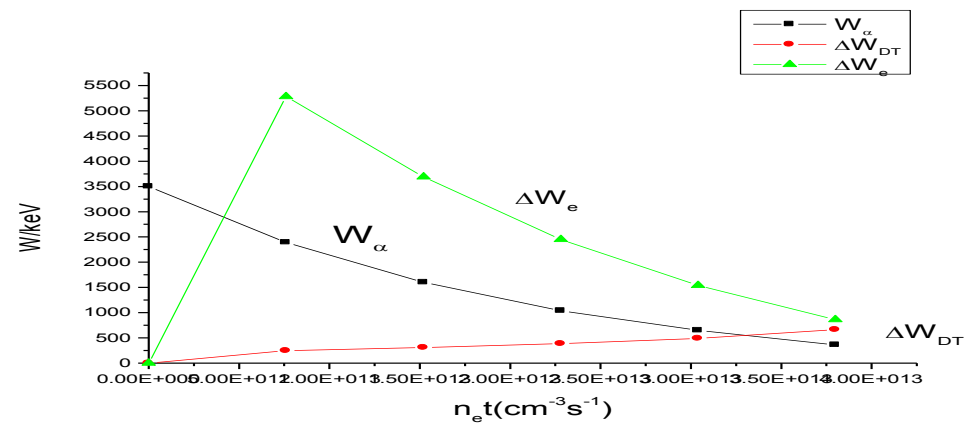

Figure 8 The Slowdown of $3.50 \mathrm{MeV}$ injected alpha particle beam $W_{\alpha}$, and the energy increment of the deuterium- tritium plasma and electrons $\Delta \mathrm{W}_{\mathrm{DT}}, \Delta \mathrm{W}_{\mathrm{e}}$ against the normalized time at $10 \mathrm{keV}$. It shows the increment in deuterium-tritium energy and the rise and fall of the electrons energy.

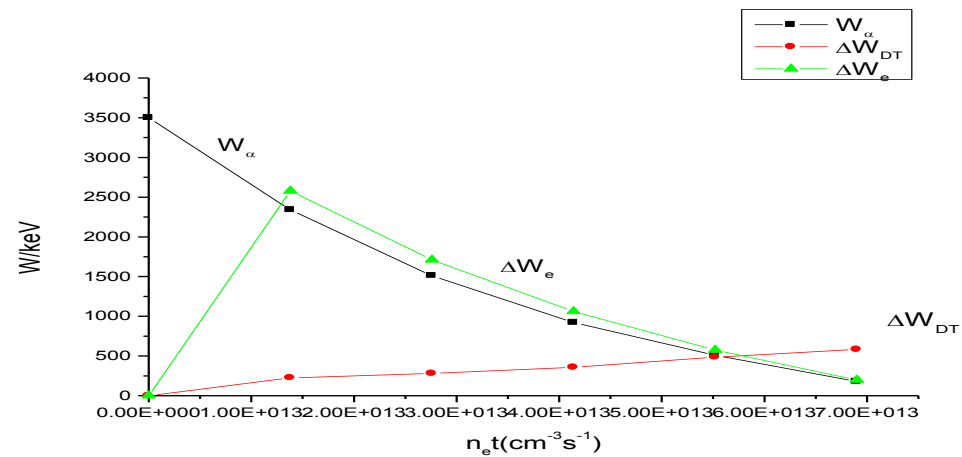

Figure 9 The Slowdown of $3.50 \mathrm{MeV}$ injected alpha particle beam $W_{\alpha}$, and the energy increment of the deuterium- tritium plasma and electrons $\Delta \mathrm{W}_{\mathrm{DT}}, \Delta \mathrm{W}_{\mathrm{e}}$ against the normalized time at $15 \mathrm{keV}$. It shows the further increment in deuterium-tritium energy and the rise and the further fall of the electrons energy.

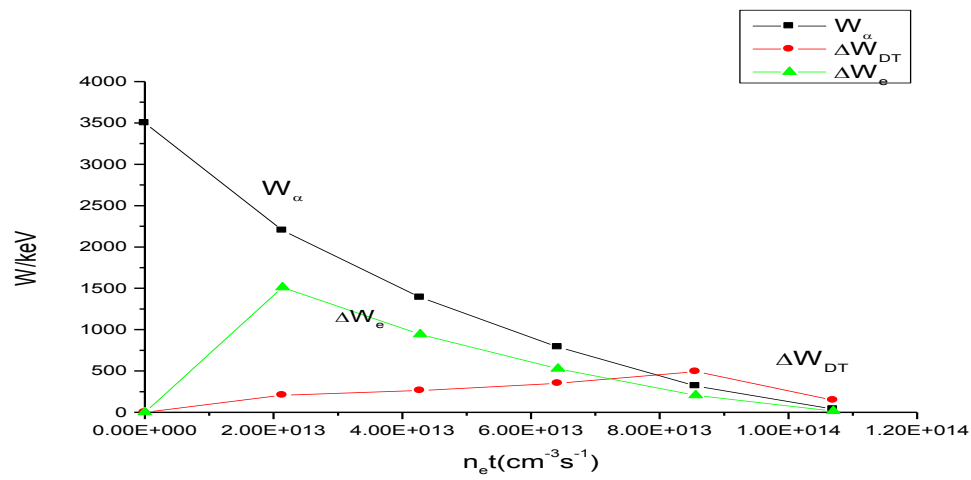

Figure 10 The Slowdown of $3.50 \mathrm{MeV}$ injected alpha particle beam $W_{\alpha}$, and the energy increment of the deuterium-tritium plasma and electrons $\Delta \mathrm{W}_{\mathrm{DT}}, \Delta \mathrm{W}_{\mathrm{e}}$ against the normalized time at $20 \mathrm{keV}$. It shows the further increment in deuterium-tritium energy and the rise and fall of the electrons energy to zero. 


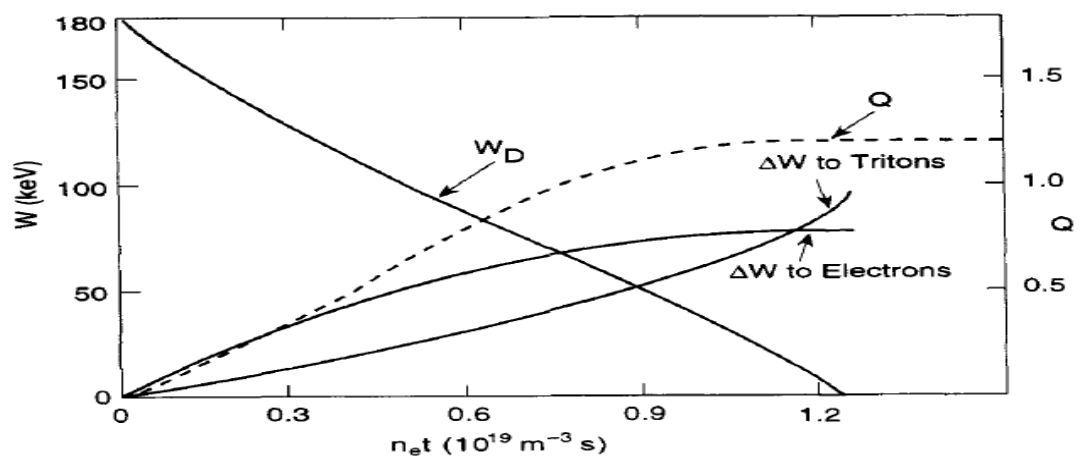

Figure 11: The Slowing down of a $180 \mathrm{keV}$ deuteron injected into a tritium plasma with $\mathrm{T}=5 \mathrm{keV}$. The energy of the deuteron is $W_{D}$ and the energy increment, $\Delta W$ are given to the Tritium and electrons in the plasma [7].

4.3 Critical Energy And Slowing Down Times For 3.5 Mev And A 180 Kev Deuterium Beam Table 1 Critical energy and Slowing Down Times for 3.5 Mev and a $180 \mathrm{KeV}$ Deuterium Beam

\begin{tabular}{|c|c|c|c|}
\hline $\mathrm{T}_{\mathrm{e}} / \mathrm{keV}$ & $\mathrm{W}_{\mathrm{c}} / \mathrm{keV}$ & $\tau_{s} / \mathrm{s}$ & $\left(W_{c} / W_{b}\right)^{3 / 2}$ \\
\hline 10 & 321.58 & 0.379 & 0.03 \\
\hline 15 & 482.38 & 0.697 & 0.05 \\
\hline 20 & 643.17 & 1.070 & 0.08 \\
\hline 5 & 71.20 & 0.268 & 0.25 \\
\hline
\end{tabular}

\section{Discussion/Conclusion}

The energetic alpha particle beam was simulated using the Fokker Planck Package FPPAC81. Fig 1-3 for the variation of the distribution functionwith normalised velocity shows that as the plasma temperature increases the population of low energy alpha particles decreases and this is consistent with Fig. 4 for the distribution obtained analytically. The graphs of energy slow down with time in Figs. 5-7 show that as the temperature of the plasma increases from $10-20 \mathrm{KeV}$ the slowdown of energy becomes more effective, this as a result of the increase in the critical energy $\mathrm{W}_{\mathrm{c}}$ which implies more energy is transferred to the ions in the plasma than the electrons. Fig 8-10 shows the variation of both the slowdown energy and the energy increment given to the plasma with the normalised time, these show that as the alpha particle slows down energy increment is given to the plasma while the plasma electrons lose energy with time Table 1 above also shows the variation critical energy and the slowing down time with temperature with both increasing. The ratio of critical energy to beam energy is seen to increase with temperature and is more for the $180 \mathrm{KeV}$ deuterium beam than for the alpha particle beam. This indicates that the rate of transfer of energy to ions in the plasma by the deuterium beam is more than that of the alpha particle beam at the same temperature.Figure11 shows the slowdown of a $180 \mathrm{KeV}$ beam in a tritium plasma at $\mathrm{T}_{\mathrm{e}}=5 \mathrm{keV}$ which compares favourably with that of Figure 8-10 for the alpha particle beam in a D-T plasma.

\section{References}

[1] McCoy, M. G., Mirin, A.A., Killeen, J., A Two-Dimensional Multispecies Nonlinear FokkerPlanck Package, Comp. Phys. Commun. 24, 37-61 (1981).

[2] Chandrasekhar, S. Review Mod. Phys. 1811 (1943).

[3] Rosenbluth, M. N., MacDonald, W. M., Judd, D. L., Phys. Rev. 107, 1 (1957).

[4]. Press, W. H., Flannery, B. P., Teukolosky, S. A. andVetterlihg, W. T., "Numerical Recipes, The art of Scientific Computing" (Cambridge Univ. Press,1992).

[5]. Ritchmeyer, R. D. and Morton, K. W., Difference methods for initial value problems (Wiley, New York, 1967).

[6] Goldston R.J. Rutherford H.P "Introduction to Plasma Physics" IOP Publishing Bristol and Philadelpia 1995.

[7] Koch R Fusion Sci. Technol. 49 No 2T, 167 (2006). 